\title{
Open-loop control of three-dimensional wakes
}

\author{
P. Poncet ${ }^{\mathrm{a}}$, G.-H. Cottet ${ }^{\mathrm{b}}$ \\ ${ }^{\text {a }}$ Dept GMM, INSA, 135 avenue de Rangueil, 31077 Toulouse Cedex 4, France \\ ${ }^{\mathrm{b}}$ LMC IMAG, Université Joseph Fourier, BP 53, 38041 Grenoble Cedex 9, France
}

\begin{abstract}
We illustrate the problem of wake optimization on the generic case of the threedimensional cylinder. After a description of the numerical method, several optimization strategies are presented, aiming at identifying vortex systems providing drag reduction or special topology of the flow. On the one hand, strategies as simple as cylinder rotation or two-dimensional stationary profiles of velocity are considered. On the other hand, an outlook of full 3D tangential controls is presented, which may be mixed or not with the simple strategies above.
\end{abstract}

Keywords : Vortex methods; 3D wakes; Hydrodynamic instabilities; 3D control; Drag reduction; Flow topology

\section{Introduction}

The control of wakes is a subject of paramount importance in aircraft and automobile industry. Depending on the particular application, wake control can have various goals and can be achieved either by passive or active strategies. Passive control mostly operates through shape optimization and often results in the addition of appendices like foilers or ribblets to the surface of the obstacle. Active control implies that one is ready to impart energy on the flow by means of actuators on the surface of the obstacle, keeping in mind that this energy must be included in the global energy budget to conclude on the efficiency of the particular control strategy.

In both these industries, shape optimization has led to important improvements in the last decades, but nowadays shows its limits, mostly due to design considerations. In the coming years, beside the need to minimize consumption, new drastic regulations in pollutants emissions will impose to explore new directions and in particular active control strategies. These strategies, beside the technology issues that they will raise, will be very demanding in terms of simulation and optimization tools. Three-dimensional wakes are still a very challenging field for simulation methods, because of the complex unsteady features of the flows. 
To illustrate active control of wakes, this paper presents in the broadest lines a robust numerical method, a high order vortex-in-cell scheme, and its application to open-loop control of three-dimensional wakes. Firstly a few simple control strategies. Secondly, the basis for full 3D control are presented, using stationary threedimensional tangential velocity distributions.

\section{Numerical method}

One considers the three-dimensional Navier-Stokes equations in a cylindrical domain $\Omega$ on its velocity-vorticity formulation :

$$
\frac{\partial \boldsymbol{\omega}}{\partial t}+(\mathbf{u} \cdot \boldsymbol{\nabla}) \boldsymbol{\omega}-(\boldsymbol{\omega} \cdot \boldsymbol{\nabla}) \mathbf{u}-\nu \Delta \boldsymbol{\omega}=0
$$

and its spanwise periodic solutions.

The present numerical approach is a lagrangian particle approximation of the vorticity $\boldsymbol{\omega}(t)$. A particle carries elements of vorticity, volumes and locations $\left(\boldsymbol{\omega}_{p}, v_{p}, \mathbf{x}_{p}\right)$, and these quantities satisfy the following system of differential equations :

$$
\frac{d \mathbf{x}_{p}}{d t}=\mathbf{u}\left(\mathbf{x}_{p}\right), \frac{d \boldsymbol{\omega}_{p}}{d t}=(\boldsymbol{\omega} \cdot \nabla \mathbf{u})\left(\mathbf{x}_{p}\right)+\nu \Delta \boldsymbol{\omega}\left(x_{p}\right)
$$

while volumes remain constant due to the incompressibility. The no-slip condition $\mathbf{u}(t)=0$ is satisfied by means of a flux of vorticity (see [4]).

Derivations are obtained by 4th-order scheme (usually centered, and biased close to walls), time integration is performed with a 4th-order Runge-Kutta step, interpolation and periodic remeshing are 3rd-order, diffusion is 2 nd order. This convection/diffusion step is followed by a flux of vorticity, enforcing the no-slip condition $\mathbf{u}(t)=0$, these two steps defining a 2nd order fractional step algorithm. Consequently, the whole algorithm solving equation (1) is globally second order. This technique has been successfully used on various two-dimensional domains and simple three-dimensional geometries (see for instance $[2,8]$ ), and more recently on cylindrical geometry (cf. $[9,10])$. This scheme may be developed in arbitrary domains using immersed boundary techniques, which has already given interesting preliminary results (see [4]).

This numerical method, taking implicitly into account transport terms, has no stability condition relying on transport, usually leading to very small time steps. Thus one can use long time steps with the present numerical method, providing an efficient tool to compute large time scales behaviour of three-dimensional flows.

Without any control (i.e. with the solid boundary condition $\mathbf{u}=0$ ), the present method recovers the main diagnostics of 2D and 3D wakes (drag and lift coefficients, Strouhal number, vorticity profiles, separation points), and the features of flow instabilities (spectral profiles, energy spectra, modes of instability, ...).

The efficiency and the stability being proved, an interesting work is to apply this tool to simulate several kinds of control strategies. 


\section{Spanwise invariant strategies}

The numerical method presented above is well-adapted for the computation of flows resulting from tangential velocities on the body, since these velocities are explicitly defined by a flux of vorticity. Computing the wake obtained by a spanwise invariant velocity (i.e. 2D control) on the body is a first step toward general three-dimensional control.

It has been shown that a body rotationally oscillating can lead to a dramatic drop of the drag coefficient, as soon as amplitude and frequency of the rotation are high enough (see [9], and also [5,11]). Beside the drag reduction, rotation forces the topology of the boundary layer and adds enstrophy to the flow, which influence the topology of the whole flow : since the body is spanwise invariant, the 3D wake comes back to its nominal two-dimensional state (see [9] and figure 2).

Such a control being costly in energy, one would like to know where this velocity on the body is the most efficient (i.e. where it has to be high). Milano and Koumoutsakos have shown in [7] that this high-efficiency area is located around the separation points. A spiked function, spanwise invariant, has thus been considered as a stationary velocity profile on the body. It turns out that this deterministic strategy leads to sharp drop of the drag coefficient, locally kills the shedding of the flow, and strongly decreases three-dimensionality (as well as body rotation). The isovorticity fields are plotted on figure 3 .

\section{Spanwise dependant profiles of velocity and outlook}

Since rotationally oscillating cylinders and stationary spiked velocity profiles both lead to drag reduction (the second kind of control being cheaper in energy), the next step toward generality is to consider three-dimensional distributions of velocity on the body. In order to limit the dimension of the control space, one considers only a few functions as control basis : one chooses the spiked 2D profile of last section multiplied by sine functions. Four functions are then chosen for the control basis : two fitting the mode A and mode B wavelengths, one between these two wavelength, and the constant function (performing drag reduction alone, as seen above).

Such functions, taken individually, forces the flow near the body, creating vortex structure of same wavelength as the control profile. The vortex structures are finally dominant in the flow (see figure 4).

First computations makes us strongly believe that combination between $2 \mathrm{D}$ and these $3 \mathrm{D}$ controls leads to more efficiency in drag reduction than the $2 \mathrm{D}$ profile, for the same energy involved. After a few complementary numerical investigations, the data obtained will be a starting set of data for genetic algorithms. Such an algorithm (cf. [6]), already successfully used to perform flow optimization (see $[1,7]$ for instance), will hopefully give interesting results for flow optimization in the four-dimensional control space described above. 


\section{References}

[1] Cottet GH, Sbalzarini I, Mueller SD, Koumoutsakos P. Optimization of trailing vortices destruction by evolution strategies. CTR Proceedings of the 2000 Summer Program.

[2] Cottet GH, Koumoutsakos P. Vortex methods, theory and practice. Cambridge University Press 2000.

[3] Cottet GH, Poncet P. Particle methods for direct numerical simulations of threedimensional wakes. J. Turbulence 2002; 3(038):1-9.

[4] Cottet GH, Poncet P. Advances in Particle-In-Cell methods for the simulation of 3D bluff-body flows. Submitted 2003.

[5] Dennis SCR, Nguyen P, Kocabiyik S. The flow induced by a rotationally oscillating and translating circular cylinder. J. Fluid Mech. 2000; 385:255-286.

[6] Hansen N, Ostermeier A. Adapting arbitrary normal mutation distribution in evolution strategies: the covariance matrix adaptation. Proceedings of the 1996 IEEE Int. Conf. on Evolutionary Computation:312-317.

[7] Milano M, Koumoutsakos P. A clustering genetic algorithm for cylinder drag optimization. J. Comput. Phys. 2002; 175(1):79-107.

[8] Ould-Sahili ML, Cottet GH, El Hamraoui M. Blending finite-differences and vortex methods for incompressible flow computations. SIAM J. Sci. Comput. 2000; 22:16551674.

[9] Poncet P. Vanishing of mode B in the wake behind a rotationnaly oscillating circular cylinder. Phys. of Fluids. 2002; 14(6):2021-2024.

[10] Poncet P. Topological aspects of the three-dimensional wake behind rotary oscillating circular cylinder. Submitted 2002.

[11] Tokumaru P, Dimotakis P. Rotary oscillation control of a cylinder wake. J. Fluid Mech. 1991; 224:77-90. 


\section{Figures}
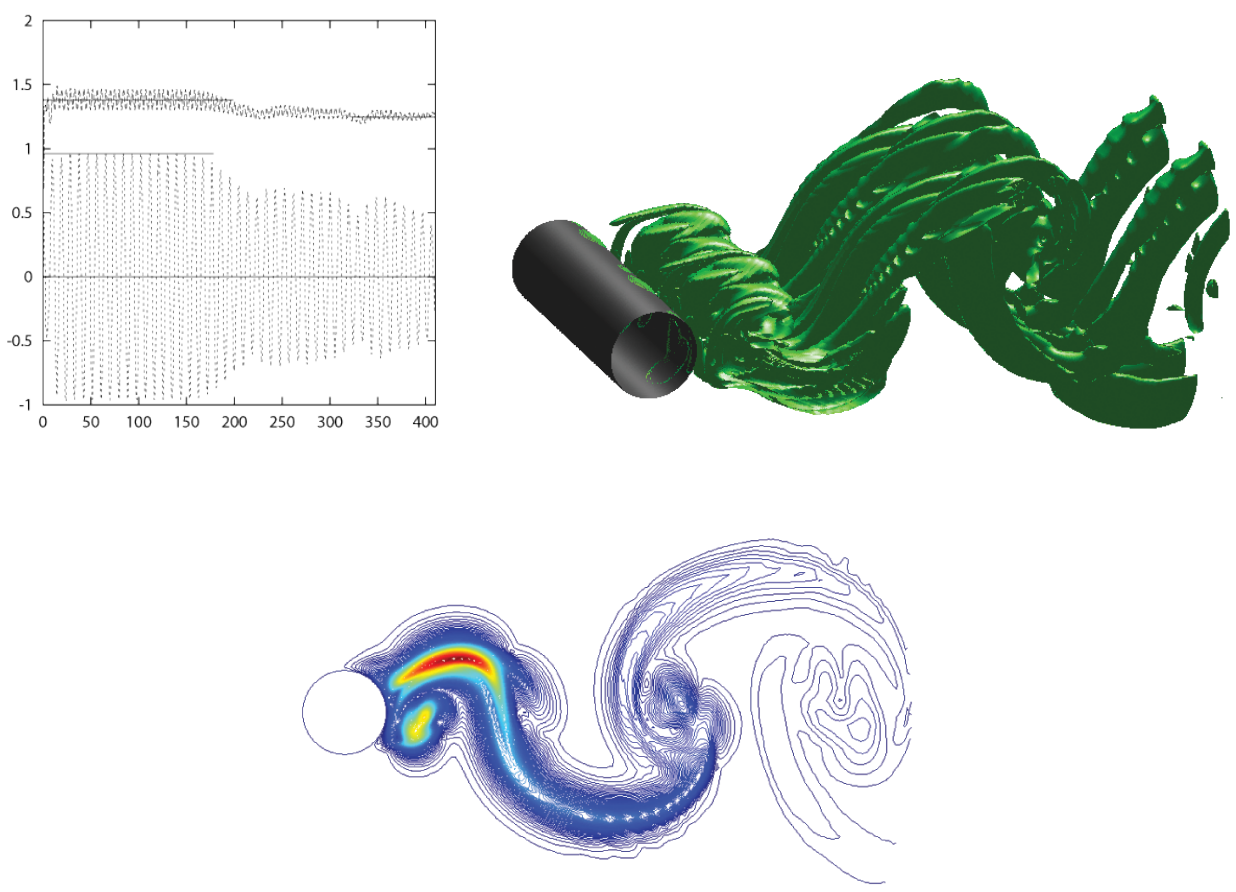

Fig. 1. Direct Numerical Simulation of an unstable 3D wake : typical drag/lift answer to three-dimensionality at $R e=300$ (top left picture), isovalue of transverse vorticity at $R e=550$ (top right picture) and lines of the spectral profile of the main wavelength at $R e=550$ (mode $\mathrm{B}$, bottom picture).
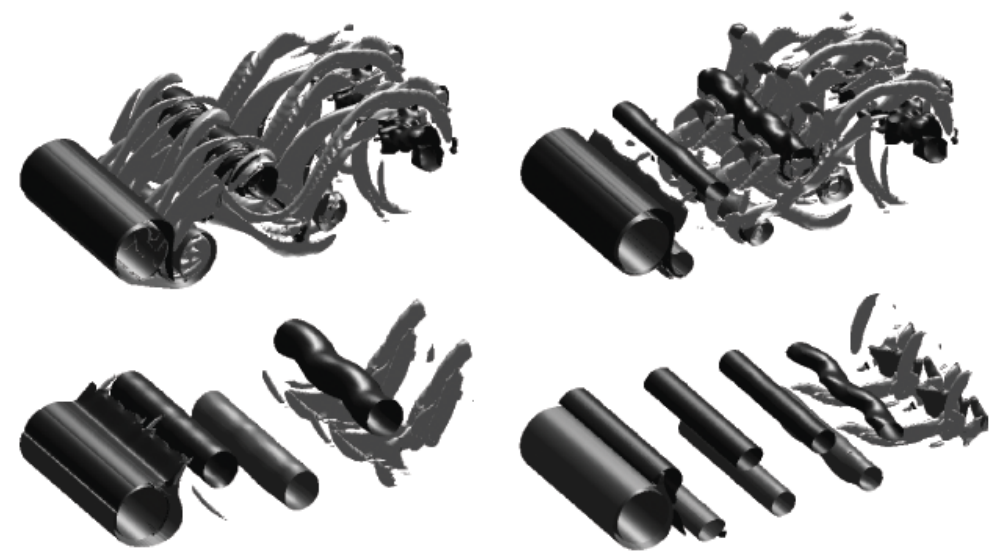

Fig. 2. Surfaces of isovorticity after activation of the rotation locked on the flow self-frequency (left pictures) and on twice this frequency (right pictures), from [9]. 


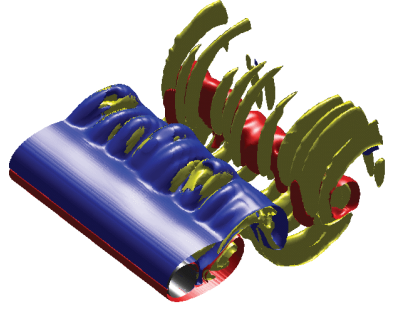

$t=270$

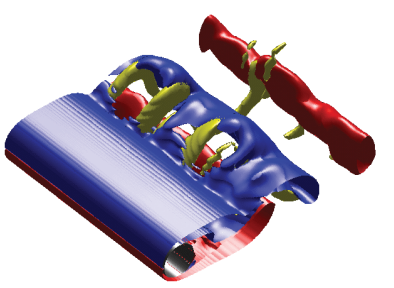

$t=290$

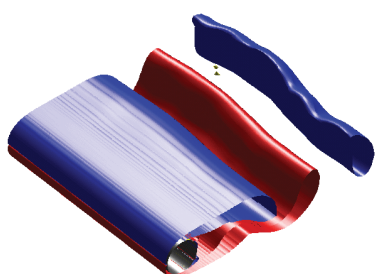

$t=310$

Fig. 3. Effect of stationary two-dimensional control : vorticity field for a sequence of times.
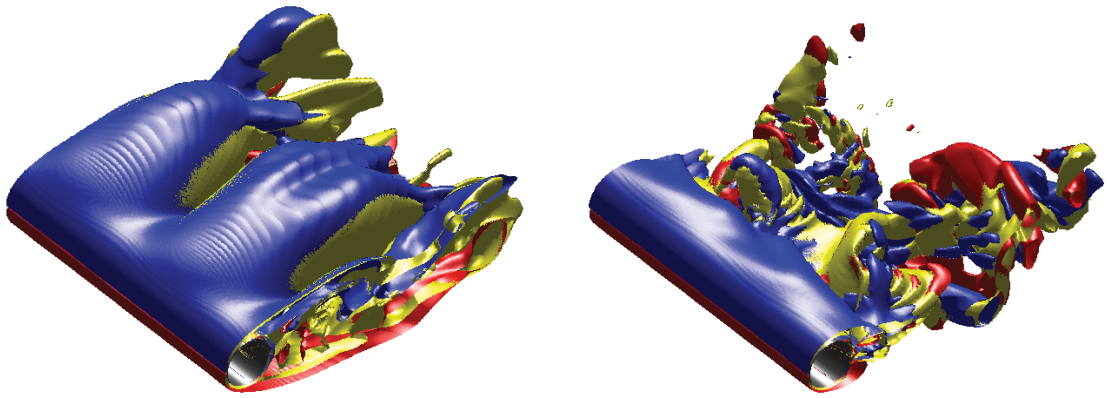

Fig. 4. Influence of control wavelength on the vorticity field, here $\lambda / D=\pi$ (fitting mode $A$ ). Left picture is $t=50$ after control activation, right picture is $t=110$. 